\title{
A POLYNOMIAL ITERATION FOR THE SPECTRAL FAMILY OF AN OPERATOR
}

\author{
by F. F. BONSALL
}

(Received 18 May, 1962)

Let $T$ be a bounded symmetric operator in a Hilbert space $H$. According to the spectral theorem, $T$ can be expressed as an integral in terms of its spectral family $\left\{E_{\lambda}\right\}$, each $E_{\lambda}$ being a certain projection which is known to be the strong limit of some sequence of polynomials in $T$. It is a natural question to ask for an explicit sequence of polynomials in $T$ that converges strongly to $E_{\lambda}$. So far as the author knows, no complete solution of this problem has been given even when $H$ has finite dimension, i.e. when $T$ is a finite symmetric matrix. Since a knowledge of the spectral family $\left\{E_{\lambda}\right\}$ of a finite symmetric matrix carries with it a knowledge of the eigenvalues and eigenvectors, a solution of the problem may have some practical value.

In a recent article [1], we gave an iterative process for constructing the projections $E_{\lambda}$, using iteration of the rational operation

$$
T \rightarrow 2 T^{2}\left(I+T^{2}\right)^{-1} \text {. }
$$

This process has certain theoretical advantages, especially for unbounded self-adjoint operators, but for computational purposes there are obvious disadvantages in the need for repeated inversion. It is true that $\left(I+T^{2}\right)^{-1}$ can be approximated by polynomials, so that we could in this way obtain explicit polynomials approximating $E_{\lambda}$. But in so doing we should lose the simplicity of the formulae, in fact we should apparently need to use polynomials of arbitrarily high degree.

In the present note we give an iterative process for constructing the projections $E_{\lambda}$ which depends only on the iteration of the cubic polynomial operation

$$
A \rightarrow A\left\{I-\frac{1}{M^{2}}(A-I)^{2}\right\} .
$$

Here $A$ is a positive symmetric operator, and $M$ is a real number, constant throughout the process.

For a heuristic understanding of the process, it is enough to consider the effect of applying the iterative process to a non-negative real number. Suppose that $M \geqq 1,0 \leqq t \leqq M$, $s=t\left[1-\frac{1}{M^{2}}(t-1)^{2}\right]$. Then it is clear that $0 \leqq s \leqq t$; and it is easily verified that if $t \geqq 1$, then $s \geqq 1$. Let $\left(t_{n}\right)$ be the sequence defined inductively by

$$
t_{1}=t, \quad t_{n+1}=t_{n}\left[1-\frac{1}{M^{2}}\left(t_{n}-1\right)^{2}\right] .
$$

Then $\left(t_{n}\right)$ is a decreasing sequence of non-negative real numbers, and if $t \geqq 1$, then $t_{n} \geqq 1$ for all $n$. The sequence $\left(t_{n}\right)$ converges to a limit $u$, which satisfies $u=u\left[1-\frac{1}{M^{2}}(u-1)^{2}\right]$, and therefore is either 0 or 1 . In fact $u=1$ whenever $t \geqq 1$, and $u=0$ whenever $t<1$. 
If we apply the same process to a mapping $f$ of a set $X$ into the real interval $[0, M]$, we see at once that the sequence of functions $f_{n}$ given by

$$
f_{1}=f, f_{n+1}=f_{n}\left[1-\frac{1}{M^{2}}\left(f_{n}-1\right)^{2}\right],
$$

converges everywhere on $X$ to the greatest characteristic function $\chi$ with $\chi \leqq f$. It is then no surprise that if we apply the process to a symmetric operator $A$ with $0 \leqq A \leqq M I$, we obtain a decreasing sequence $\left(A_{n}\right)$ of operators that converges strongly to the greatest projection $Q$ that is permutable with $A$ and satisfies $Q \leqq A$. To obtain the other projections in the spectral family, we replace $A$ by a scalar multiple of $A$. To find all the projections in the spectral family of a bounded symmetric operator $T$, not necessarily positive, we take

$$
A=(\beta-\lambda)^{-1}(\beta I-T),
$$

where $\beta$ is an upper bound for $T$. In general, the sequence $\left(A_{n}\right)$ converges strongly to $Q$, i.e. $\lim _{n \rightarrow \infty} A_{n} x=Q x$ for all $x$ in $H$. But if the operator $A$ is compact, then the convergence is uniform, i.e. $\left(A_{n}\right)$ converges to $Q$ with respect to the operator norm.

A statement is given in [1] of the very few elementary properties of operators in Hilbert space that we need, all of which are proved in [2, pp. 259-265]. In particular, we do not need the spectral theorem; and therefore, as in [1], we obtain an elementary proof of that much proved theorem.

Lemma. Let $M \geqq 1$, let $A$ be a bounded symmetric operator in $H$ such that $0 \leqq A \leqq M I$, and let $B=A\left[I-\frac{1}{M^{2}}(A-I)^{2}\right]$. Then

(i) $0 \leqq B \leqq A$, $P \leqq B$.

(ii) every projection $P$ which is permutable with $A$ and which satisfies $P \leqq A$, also satisfies

Proof. (i) $A-B=\frac{1}{M^{2}} A(A-I)^{2} \geqq 0$,

and

$$
B=\frac{1}{M^{2}} A[(M+1) I-A][(M-1) I+A] \geqq 0 .
$$

(ii) Let $P$ be a projection permutable with $A$ which satisfies $P \leqq A$. Since $P^{2}=P$ and all the operators concerned commute, we have

Therefore

$$
P B=P A-\frac{1}{M^{2}} A(P A-P)^{2} .
$$

$$
P B-P=(P A-P)\left[I-\frac{1}{M^{2}} A(P A-P)\right] \text {. }
$$

We have $0 \leqq P \leqq I$, and so $A(P A-P) \leqq A^{2} \leqq M^{2} I$. Since also

$$
P A-P=P(A-P) \geqq 0,
$$

it follows that $P B-P \geqq 0$. Finally, $B \geqq P B \geqq P$. 
THEOREM 1. Let $M \geqq 1$, and let $A$ be a bounded symmetric operator in $H$ such that $0 \leqq A \leqq M I$. Let the sequence $\left(A_{n}\right)$ be defined inductively by

Then (i) $0 \leqq A_{n+1} \leqq A_{n} \quad(n=1,2, \ldots)$,

$$
A_{1}=A, \quad A_{n+1}=A_{n}\left[I-\frac{1}{M^{2}}\left(A_{n}-I\right)^{2}\right] .
$$

(ii) the sequence $\left(A_{n}\right)$ converges strongly to a projection $Q$,

(iii) $Q$ commutes with $A$ and satisfies $Q \leqq A$,

(iv) $Q$ is maximal in the sense that if $P$ is a projection permutable with $A$ and satisfying $P \leqq A$, then $P \leqq Q$,

(v) $(I-A)(I-Q) \geqq 0$.

Proof. (i) This follows at once from the lemma.

(ii) and (iii) It follows from (i) that the sequence $\left(A_{n}\right)$ converges strongly to a bounded symmetric operator $Q$, and that $0 \leqq Q \leqq A$. Since the operators $A_{n}$ commute with $A$, so does $Q$. It remains to prove that $Q^{2}=Q$. We have $\left\|A_{n}\right\| \leqq\|A\|$, and therefore the sequences $\left(A_{n}^{2}\right)$ and $\left(A_{n}^{3}\right)$ converge strongly to $Q^{2}$ and $Q^{3}$ respectively. Therefore

$$
Q=Q\left[I-\frac{1}{M^{2}}(Q-I)^{2}\right], \quad[Q(Q-I)]^{2}=0 .
$$

Since $Q(Q-I)$ is symmetric, this implies that $Q(Q-I)=0$, i.e. $Q^{2}=Q$.

(iv) If $P$ is a projection that commutes with $A$ and satisfies $P \leqq A$, then, by the lemma, we have $P \leqq A_{n}$ for all $n$. Therefore $P \leqq Q$.

(v) We have

and

$$
I-A_{n+1}=\left(I-A_{n}\right)\left[I+\frac{1}{M^{2}} A_{n}\left(I-A_{n}\right)\right],
$$

$$
I+\frac{1}{M^{2}} A_{n}\left(I-A_{n}\right) \geqq 0 .
$$

It follows from this, by induction, that

Therefore

$$
(I-A)\left(I-A_{n}\right) \geqq 0 \quad(n=1,2, \ldots) .
$$

THEOREM 2. Let $T$ be a bounded symmetric operator in $H$, and let $\alpha, \beta$ be real numbers such that $\alpha I \leqq T \leqq \beta I$. For each $\lambda<\beta$, let $T_{\lambda}$ be defined by $T_{\lambda}=(\beta-\lambda)^{-1}(\beta I-T)$, and let $M_{\lambda}$ be chosen so that $M_{\lambda} \geqq 1$ and $M_{\lambda} \geqq(\beta-\lambda)^{-1}(\beta-\alpha)$. Let $E_{\beta}=I$, and for each $\lambda<\beta$, let $E_{\lambda}$ be the projection $Q$ constructed as in Theorem 1 with $A=T_{\lambda}$ and $M=M_{\lambda}$. Then $\left\{E_{\lambda}: \lambda \leqq \beta\right\}$ is the spectral family for $T$, i.e.

(i) the projections $E_{\lambda}$ commute with $T$ and with each other;

(ii) $E_{\lambda}=0 \quad(\lambda<\alpha)$;

(iii) $E_{\lambda} \leqq E_{\mu} \quad(\lambda<\mu \leqq \beta)$;

(iv) $\lambda\left(E_{\mu}-E_{\lambda}\right) \leqq T\left(E_{\mu}-E_{\lambda}\right) \leqq \mu\left(E_{\mu}-E_{\lambda}\right) \quad(\lambda<\mu \leqq \beta)$;

(v) the family $\left\{E_{\lambda}: \lambda \leqq \beta\right\}$ is strongly continuous on the right. 
Proof. (i) For each $\lambda<\beta, T_{\lambda}$ satisfies the inequalities $0 \leqq T_{\lambda} \leqq M_{\lambda} I$. Thus Theorem 1 is applicable, and yields the projections $E_{\lambda}$ that satisfy (i).

(ii) If $E_{\lambda} \neq 0$, there exists $x_{0} \neq 0$ with $E_{\lambda} x_{0}=x_{0}$. But then

However

$$
\left(T_{\lambda} x_{0}, x_{0}\right) \geqq\left(E_{\lambda} x_{0}, x_{0}\right)=\left(x_{0}, x_{0}\right)>0 \text {. }
$$

$$
\left(T_{\lambda} x, x\right)=(\beta-\lambda)^{-1}[\beta(x, x)-(T x, x)] \leqq(\beta-\alpha)(\beta-\lambda)^{-1}(x, x),
$$

and so $\lambda<\alpha$ implies $E_{\lambda}=0$.

(iii) We have

$$
E_{\lambda} \leqq T_{\lambda} \leqq T_{\mu} \quad(\lambda<\mu<\beta)
$$

and $E_{\lambda}$ is a projection permutable with $T_{\mu}$. Therefore, by the maximal property of $E_{\mu}, E_{\lambda} \leqq E_{\mu}$. Also $E_{\lambda} \leqq I=E_{\beta}$.

(iv) Let $\lambda<\mu \leqq \beta$. We have $T_{\mu} \geqq E_{\mu}$ whenever $\mu<\beta$, and $T \leqq \beta I$. Therefore

$$
T \leqq \beta\left(I-E_{\mu}\right)+\mu E_{\mu} \text {. }
$$

By (iii), $E_{\mu}-E_{\lambda} \geqq 0$, and $E_{\mu}\left(E_{\mu}-E_{\lambda}\right)=E_{\mu}-E_{\lambda}$. Therefore

$$
T\left(E_{\mu}-E_{\lambda}\right) \leqq \mu\left(E_{\mu}-E_{\lambda}\right) .
$$

Also $\left(I-T_{\lambda}\right)\left(I-E_{\lambda}\right) \geqq 0$, i.e. $\lambda\left(I-E_{\lambda}\right) \leqq T\left(I-E_{\lambda}\right)$. Multiplication of this inequality by $E_{\mu}$ yields

$$
\lambda\left(E_{\mu}-E_{\lambda}\right) \leqq T\left(E_{\mu}-E_{\lambda}\right) .
$$

(v) To prove that the family $\left\{E_{\lambda}: \lambda \leqq \beta\right\}$ is strongly continuous on the right, it is enough to show that the sequence $\left(E_{\lambda_{n}}\right)$ converges strongly to $E_{\lambda}$ whenever $\left(\lambda_{n}\right)$ is a decreasing sequence that converges to $\lambda$ and satisfies

$$
\lambda<\lambda_{n}<\beta \quad(n=1,2, \ldots) .
$$

If $\left(\lambda_{n}\right)$ is such a sequence, then, by (iii),

$$
E_{\lambda} \leqq E_{\lambda_{n+1}} \leqq E_{\lambda_{n}} \quad(n=1,2, \ldots) .
$$

Therefore $\left(E_{\lambda_{n}}\right)$ converges strongly to a projection $P$, and

Thus

$$
E_{\lambda} \leqq P \leqq E_{\lambda_{n}} \leqq T_{\lambda_{n}} \quad(n=1,2, \ldots)
$$

$$
E_{\lambda} \leqq P \leqq T_{\lambda},
$$

and, by the maximal property of $E_{\lambda}$, we have $E_{\lambda}=P$.

THEOREM 3. Let $A$ be a compact positive operator in $H$, and let $\left(A_{n}\right)$ and $Q$ be the corresponding sequence and projection defined as in Theorem 1 . Then $\left(A_{n}\right)$ converges to $Q$ in operator norm.

Proof. It is easily seen, by induction, that

$$
A_{n}=B_{n} A \quad(n=1,2, \ldots),
$$

where $B_{n}$ is a polynomial in $A$, and 


$$
0 \leqq B_{n+1} \leqq B_{n} \quad(n=1,2, \ldots) .
$$

The sequence $\left(B_{n}\right)$ therefore converges strongly to a positive operator $B$ permutable with $A$. Since $A_{n}=B_{n} A$, and $\left(A_{n}\right)$ converges strongly to $Q$, we have $Q=B A$. We have

$$
0 \leqq\left(B_{n+1}-B\right)^{2} \leqq\left(B_{n}-B\right)^{2} \quad(n=1,2, \ldots),
$$

and so $\left\|\left(B_{n}-B\right) x\right\|$ converges decreasingly to zero as $n \rightarrow \infty$ for each $x$ in $H$. Let $K$ be the unit ball in the Hilbert space $H$, and let $X$ be the closure of $A K$ in the norm topology. Since $A$ is a compact operator, $X$ is a compact set in the norm topology. By Dini's theorem, it follows that $\lim _{n \rightarrow \infty}\left\|\left(B_{n}-B\right) x\right\|=0$ uniformly on $X$. Therefore $\lim _{n \rightarrow \infty}\left\|\left(B_{n}-B\right) A x\right\|=0$ uniformly on $K$, and so $\lim _{n \rightarrow \infty}\left\|A_{n}-Q\right\|=0$.

\section{REFERENCES}

1. F. F. Bonsall, A formula for the spectral family of an operator, J. London Math. Soc. 35 (1960), 321-333.

2. F. Riesz and B. Sz.-Nagy, Leçons d'Analyse Fonctionelle (Budapest, 1952).

\section{King's College}

DURHAM UNIVERSITY

NEWCASTLE UPON TYNE 